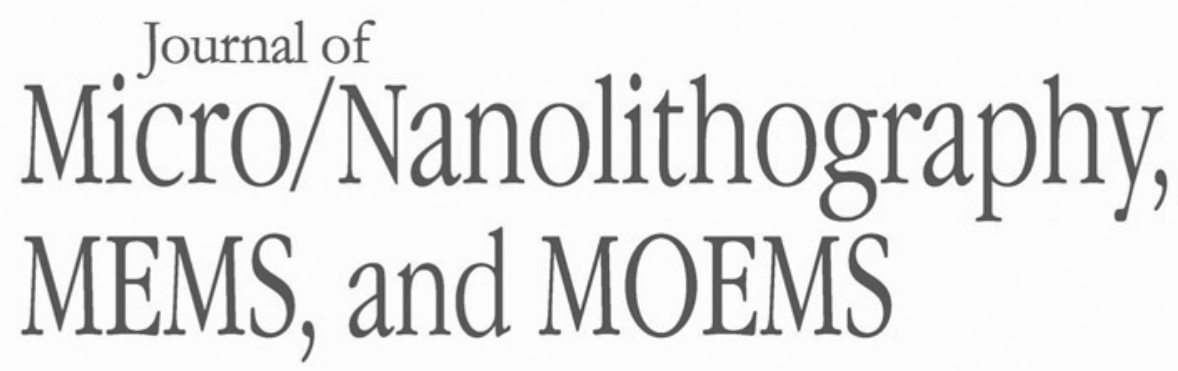

Nanolithography.SPIEDigitalLibrary.org

\title{
Tip-on-tip imaging and self-consistent calibration for critical dimension atomic force microscopy: refinements and extension to second lateral axis
}

\author{
Ronald Dixson
}




\title{
Tip-on-tip imaging and self-consistent calibration for critical dimension atomic force microscopy: refinements and extension to second lateral axis
}

\author{
Ronald Dixson* \\ National Institute of Standards and Technology, Microsystems and Nanotechnology Division, Gaithersburg, Maryland, United States
}

\begin{abstract}
One type of atomic force microscopy (AFM) used for critical dimension (CD) metrology is commonly referred to as CD-AFM; it uses flared tips and two-dimensional (2-D) surface sensing to enable scanning of features with near-vertical sidewalls. An important consideration in this type of CD-AFM metrology is the calibration uncertainty of the tip width (TW). Standards for traceable TW calibration have thus been developed both by National Metrology Institutes and commercial suppliers. The National Institute of Standards and Technology has previously reported the implementation of a self-consistency TW calibration using three CD-AFM tips to image each other. The results of this method were shown to be consistent with prior calibrations based on transmission electron microscope cross sections. The extension of this method to tips $<50 \mathrm{~nm}$ is demonstrated, as well as the extension of the method to include a second lateral axis. @ 2018 Society of Photo-Optical Instrumentation Engineers (SPIE) [DOI: 10.1117/1.JMM.17.4.044001]

Keywords: critical dimension atomic force microscopy; self-consistent calibration; linewidth; metrology.

Paper 18091 received Jul. 26, 2018; accepted for publication Oct. 3, 2018; published online Oct. 19, 2018.
\end{abstract}

\section{Introduction}

One type of atomic force microscopy (AFM) used for critical dimension $(\mathrm{CD})$ metrology is commonly referred to as $\mathrm{CD}$ $\mathrm{AFM}$; it uses flared tips and two-dimensional (2-D) surface sensing to enable scanning of features with near-vertical sidewalls. ${ }^{1}$ An important consideration in this type of CDAFM metrology is the calibration uncertainty of the tip width (TW). Standards for traceable TW calibration have thus been developed both by National Metrology Institutes and commercial suppliers. ${ }^{2-4}$ The National Institute of Standards and Technology (NIST) has been working with the flared-tip form of CD-AFM technology since the early 2000 s with the goals of developing methods and standards for traceable CD-AFM metrology.

It is important to note that there are alternative AFM technologies that can perform CD metrology. Specifically, the last decade has witnessed the development and proliferation of a tilting-head AFM technique, which is capable of scanning vertical sidewalls using conventional AFM tips. ${ }^{5}$ This technology has gained increasing acceptance within nanomanufacturing metrology for both $\mathrm{CD}$ metrology and sidewall roughness measurements. ${ }^{6}$ At the present time, however, NIST does not have experience with tilting-head AFM technology. The present work thus involves exclusively the flared-tip form of CD-AFM.

Previously, NIST has reported the implementation of a self-consistency TW calibration using three CD-AFM tips to image each other. ${ }^{7}$ The results of this method were shown to be consistent with prior calibrations based on transmission electron microscope cross-sections. However, tips $<50 \mathrm{~nm}$ were not used successfully, and the metrology was only performed in a single lateral axis: the fast scan axis. In the present work, the extension of this method to

*Address all correspondence to: Ronald Dixson, E-mail: ronald.dixson@nist .gov tips $<50 \mathrm{~nm}$ is demonstrated, as well as the extension of the method to include a second lateral axis: the slow scan axis.

\section{Concept of Self-Consistent Calibration}

At the simplest level, the basic concept of this approach is to use three different CD-AFM tips to image each other and to measure the apparent total width of each tip-on-tip pair. There are thus three equations and three unknowns, allowing for a unique solution of the three TWs. The concept of this method is shown in Fig. 1, and it leads to the following equations for the three TWs:

$$
\begin{aligned}
& \mathrm{TW}_{A}=\frac{1}{2}\left[W_{\mathrm{AB}}-W_{\mathrm{BC}}+W_{\mathrm{AC}}\right], \\
& \mathrm{TW}_{B}=\frac{1}{2}\left[W_{\mathrm{AB}}+W_{\mathrm{BC}}-W_{\mathrm{AC}}\right], \\
& \mathrm{TW}_{C}=\frac{1}{2}\left[W_{\mathrm{AC}}+W_{\mathrm{BC}}-W_{\mathrm{AB}}\right],
\end{aligned}
$$

where $\mathrm{TW}_{A}$ is the width of tip $\mathrm{A}, W_{A B}$ is the apparent width of tip A imaging tip B, and similarly for the other tips and combinations. Note that for experiments in which the vertical edge height (VEH) of the tips is of interest, an analogous set of equations can be developed.

As previously pointed out by Eves and Green, ${ }^{8}$ such a simple analytical description is only strictly applicable with the tips for which a well-defined width metric is possible, for example, spherical tips having well-defined radii. Since Eves and Green worked with conventional conical AFM tips, their analysis was extended and incorporated

$1932-5150 / 2018 / \$ 25.00$ C 2018 SPIE 

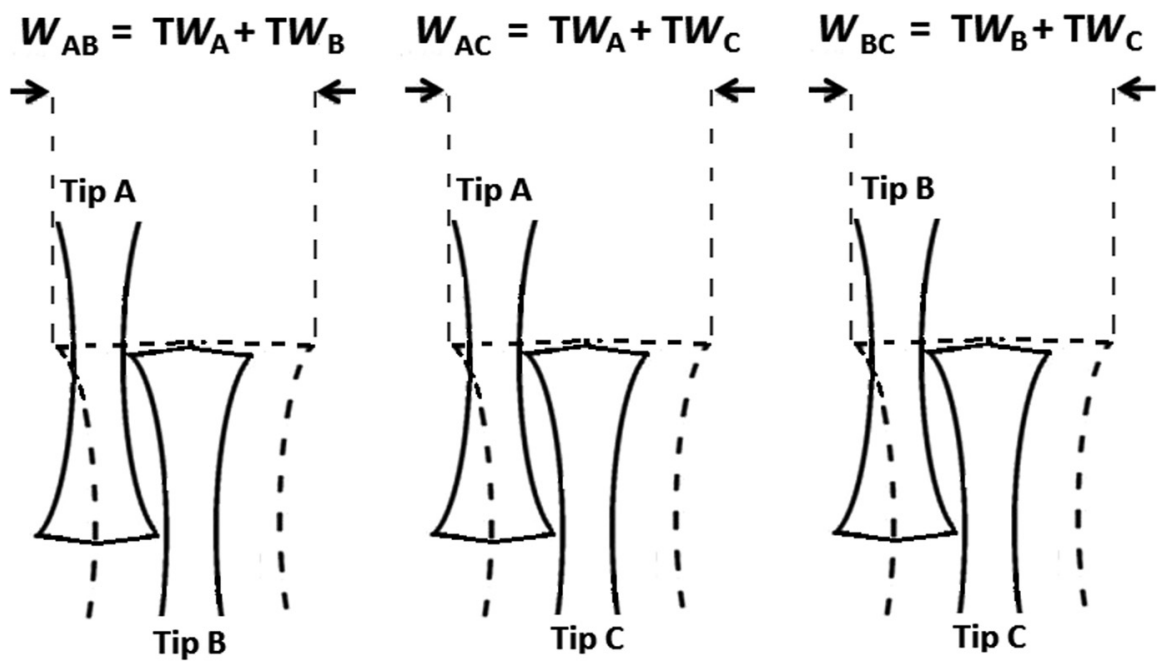

Fig. 1 Schematic illustration of tip-on-tip imaging and three tip self-consistency calibration. Note that a two-tip experiment would be insufficient because the number of independent constraints must be equal to the number of tips, and tip $A$ imaging tip $B$ is equivalent to tip $B$ imaging tip $A$.

mathematical morphology to allow the extraction of selfconsistent profiles of tip shape along the fast scan axis.

As CD-AFM tips are designed to measure near-vertical sidewalls, the geometry of most CD-AFM tips is generally favorable for the use of simplifying assumptions, and the analytical approximation of three equations and three unknowns is often applicable. Strictly speaking, there is no single value of CD-AFM TW. However, for tips in good condition, the dilation effect of the tip on a feature can be very well approximated by the zeroth-order TW. This is essentially the lateral separation of the flare apices at the contact points or points of greatest extent in the fast scan axis. For tip-on-tip imaging, this TW metric is readily extracted from the images, and it can be treated additively following the analytical model given by Eq. (1) through Eq. (3).

\section{Experimental Implementation}

NIST has a CD-AFM, used for the present work, that has been characterized and calibrated to perform traceable measurements of width, pitch, and height. ${ }^{9}$ It is utilized both to support other NIST projects and to provide measurements for other organizations. The details of operation and calibration of this system will be mentioned as appropriate.

Although conventional AFM tips are available from a wide range of commercial suppliers, there are relatively few suppliers of CD-AFM tips. One consequence of this is that there are generally accepted conventions and nomenclature to describe CD-AFM tips with respect to functional characteristics. For example, the designation critical dimension round (CDR) is normally used to designate a flared CD tip having approximate rotational symmetry in the $z$-axis. A numerical designation typically follows, as in CDR50 to specify the nominal width in nanometers. Additional characters are sometimes used to specify other characteristics such as coating or material composition. This paper will follow these conventions with additional explanation where appropriate.

As was the case for the prior NIST work, the reference method of tip calibration was the conventional method involving two types of tip characterizers: a vertical parallel structure (VPS) to calibrate TW, and a flared silicon ridge
(FSR) to calibrate VEH. Additionally, traceability of the TW is achieved using the NIST single-crystal critical dimension reference material (SCCDRM) ${ }^{3,10}$ to calibrate the VPS. The expanded uncertainty for the conventional method of TW calibration is typically $1 \mathrm{~nm}(k=2)$ to $1.3 \mathrm{~nm}$ $(k=2)$. There is presently no traceable standard for $\mathrm{VEH}$, but the expanded uncertainty of the conventional method for VEH measurement is thought to be $\sim 7 \mathrm{~nm}(k=2)$. The uncertainties of both the conventional and self-consistency methods of tip calibration are discussed in more detail in Sec. 4. Note that the remainder of Sec. 3 is thus limited in scope to the description of the tip-on-tip self-consistency results and comparison with the conventional results, and all treatment and discussion of uncertainty are deferred to Sec. 4.

\subsection{Tip-on-Tip Imaging}

Generally, the major challenges to tip-on-tip imaging are navigation and the transfer of mechanical energy from the imaging tip and cantilever system to the target tip and cantilever. However, these turn out to be less daunting than might be expected.

These challenges are potentially dependent upon the mounting of the target tip for imaging. Initially, elaborate holder designs were considered, but the dimensional variation from cantilever to cantilever ultimately suggested that this would be impractical. The method used in the prior NIST work was to mount the tips on a 200-mm silicon wafer using double-sided carbon tape. ${ }^{7}$ For the current work, an aluminum substrate was also used. This carrier has a recessed slot machined into it with a 3-deg surface slope on the bottom. The function of this slope is to adjust the angle of observation of the tip under imaging. Figure 2 shows a top down photographs of standard AFM tip substrates mounted on both carriers with double-sided carbon tape.

The CD-AFM operated by NIST has a tip mount that induces a 3-deg tilt of the cantilever, along its long axis, with respect to the imaged surface. When scanning along this axis, the apparent tip geometry is rotated by this tilt. Partly for this reason, the instrument fast-scan axis normally 


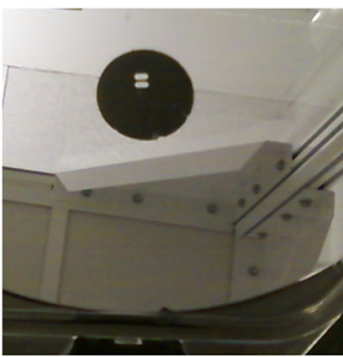

(a)

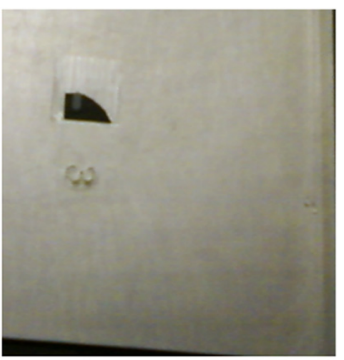

(b)
Fig. 2 Top down photographs of standard AFM tip substrates mounted with double-sided carbon tape on (a) the 200-mm Si wafer and (b) the Al carrier with recessed slot machined at 3-deg slope.

coincides with the short axis of the cantilever-which is not tilted. Some cantilevers are available with incorporated 3-deg tilt of the tip with respect to the cantilever to compensate for the tip mounting angle in the instrument. Generally, the most effective way to mount nontilt-compensated tips is horizontally on the silicon wafer, and the most appropriate way to mount tilt-compensated tips is using the aluminum carrier. For tilt-compensated tips, it is thus possible to align the axes of both tips with each other and with the $z$-axis of the instrument.

Navigation to achieve tip-on-tip contact is the most challenging for very small tips. The tips must ultimately contact each other at their extrema; if the initial contact between the tips is too far down on their respective shanks, then the available $z$-range of the scanner will not be sufficient to achieve tip overlap using just a single engage. Thus, the probability of success on initial engage is a function of navigation optics, stage accuracy, $z$-axis scanner range, and operator experience. In the case of the NIST experiments, it was not typically possible to achieve tip overlap using just a single engage. However, it was usually possible to readily tune up on the overlap by using a repeated cycle of tip engage, disengage, and adjustment of lateral scanner offsets.

\subsection{Refined Experiments Using a Single Lateral Axis}

The prior NIST work involved three types of CD tips: CDR50Cs, CDR300s, and CDR850s. Subsequently, for the present work, experiments have been performed using the following tip types: CDR50S, CDR140, and EBDCDR25. An experiment was also performed combining a CDR120 and CDR300 and substituting a linewidth standard for the third tip.

CDR50S tips have a shorter effective length than CDR50C tips, and thus have greater lateral stiffness. So it was expected that CDR50S tips would exhibit better selfconsistent calibration performance than did CDR50C tips. This was generally the case, but the performance did not reach the level previously observed using CDR300 tips.

An example tip-on-tip image using CDR50S tips is shown in Fig. 3. The image stability is good. For the measurements using CDR50S tips, the instrument was operated using fast dither tube actuation (FDTA) mode. For purposes of this work, a consequence of using FDTA is that this is generally expected to minimize additional lateral tip motion and mechanical coupling between the target and imaging tips.

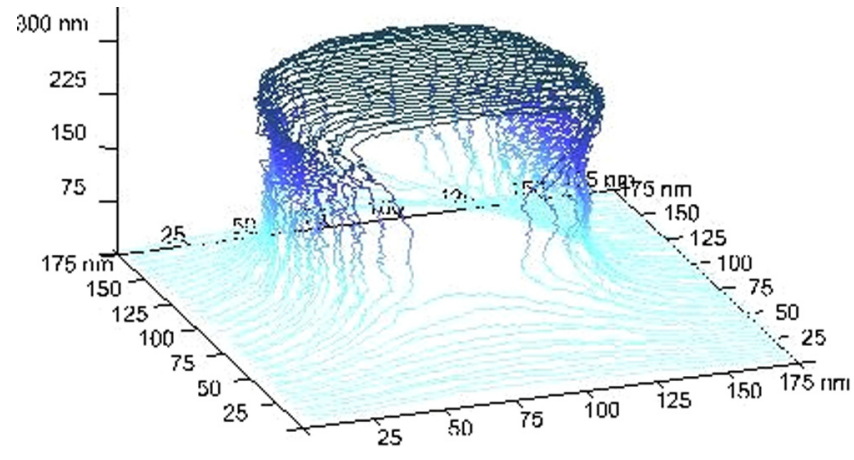

Fig. 3 Tip-on-tip image using CDR50S tips. The imaging stability is better than previously observed using CDR50C tips. FDTA mode is used.

The results of the self-consistency analysis using the CDR50S data are shown in Table 1. A refinement that has been made to the analysis in the current work is to separately track the "left" and "right" values of the VEH for each tip. As in the prior work, results are included using both the functionality of the native CD-AFM software and an off-line analysis developed at NIST. The offline analysis offers the advantages of not depending on manual or visual analysis of the data and it removes user judgement in identifying the relevant extrema. It is also potentially advantageous for tips that exhibit significantly nonideal shapes-such as those for which the lateral extrema occur in different scan lines. On the other hand, the use of the native software provides very high convenience to an end user. A typical end user working with ordinary CD-AFM tips would probably derive minimal benefit from the additional effort of offline analysis, but it is useful to NIST both as a validating comparison, and it supports the NIST goal of developing a traceable VEH calibration.

Generally, the level of agreement is significantly better than previously observed using CDR50C tips. With CDR50C tips, TW differences of 6 to $8 \mathrm{~nm}$ were observed

Table 1 Results of conventional tip calibration and self-consistency method for CDR50S tips.

\begin{tabular}{|c|c|c|c|c|c|c|}
\hline \multirow{2}{*}{\multicolumn{2}{|c|}{ Tip }} & \multicolumn{5}{|c|}{ Measurement method } \\
\hline & & \multicolumn{2}{|c|}{$\begin{array}{l}\text { Conventional } \\
\text { (VPS/FSR) }\end{array}$} & \multicolumn{3}{|c|}{$\begin{array}{c}\text { Tip-imaging self- } \\
\text { consistency }\end{array}$} \\
\hline \multirow[b]{2}{*}{ Type } & \multirow[b]{2}{*}{ Specifier } & \multirow{2}{*}{$\begin{array}{l}\text { TW } \\
(\mathrm{nm})\end{array}$} & \multirow{2}{*}{$\frac{\text { VEH (nm) }}{\text { (Left, right) }}$} & \multirow{2}{*}{$\begin{array}{l}\text { Analysis } \\
\text { software }\end{array}$} & \multirow{2}{*}{$\begin{array}{l}\text { TW } \\
(\mathrm{nm})\end{array}$} & \multirow{2}{*}{$\frac{\text { VEH (nm) }}{\text { (Left, right) }}$} \\
\hline & & & & & & \\
\hline \multirow[t]{6}{*}{ CDR50S } & A & 57.4 & $18.5,20.6$ & Native & 57.0 & $21.9,18.9$ \\
\hline & & & & Off-line & 59.3 & $22.9,25.2$ \\
\hline & B & 61.8 & $16.2,19.8$ & Native & 56.2 & $20.8,15.9$ \\
\hline & & & & Off-line & 58.6 & $29.7,22.4$ \\
\hline & C & 61.1 & $19.2,22.8$ & Native & 58.3 & $15.0,21.8$ \\
\hline & & & & Off-line & 58.6 & $21.6,20.2$ \\
\hline
\end{tabular}




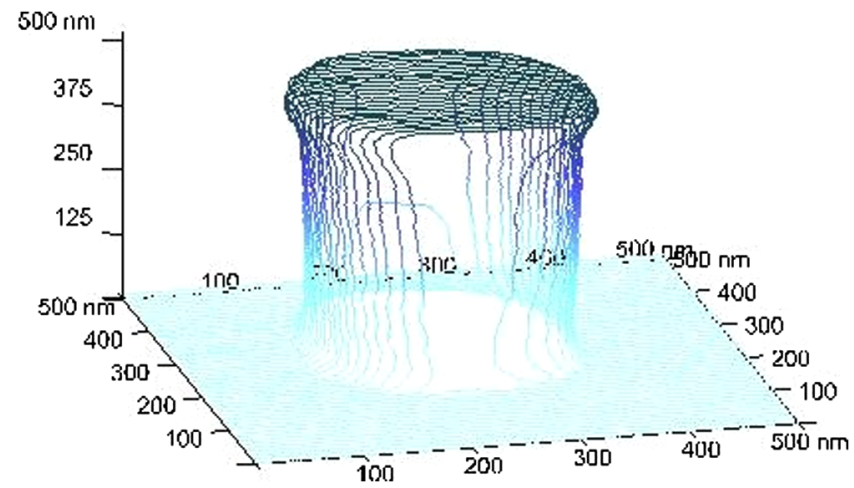

Fig. 4 Tip-on-tip image using CDR140 tips. The imaging stability is good. FDTA mode is used.

between the conventional calibration and the self-consistency method. In the present case of CDR50S tips, the TW agreement is generally at the 2- to 4-nm level. The agreement among the VEH values is also generally better, though not dramatically. Despite the better results obtained using CDR50S tips in this experiment, the performance still does not reach the level previously observed using CDR300 tips.

The most successful run thus far was accomplished using CDR140 tips. An example tip-on-tip image is shown in Fig. 4, and the results of the self-consistency experiment are shown in Table 2. As was the case for the CDR50S results shown above, the instrument was operated using the FDTA mode for the CDR140 measurements.

There is very good agreement between the self-consistency linewidth results and the conventional method-essentially equivalent or surpassing the prior level using CDR300 tips. The agreement between the offline and native analyses is slightly better. Although the VEH results show more variation and differences, when left/right averages are considered, the VEH results also slightly surpass those for the CDR300 tips.

The previous work of Eves and Green ${ }^{8}$ using conventional AFM tips also substituted a linewidth standard for the third

Table 2 Results of conventional tip calibration and self-consistency method for CDR140 tips.

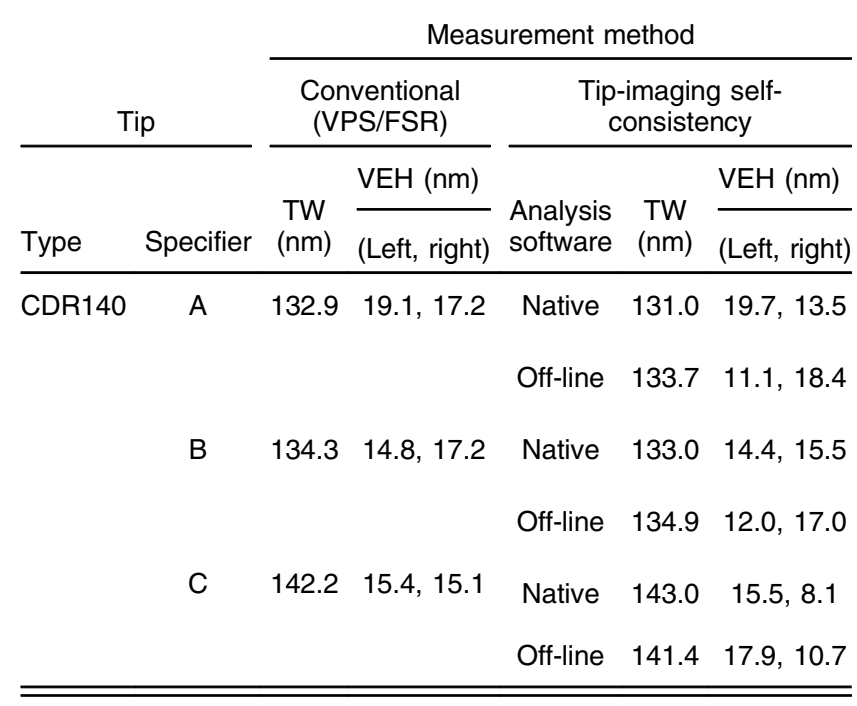

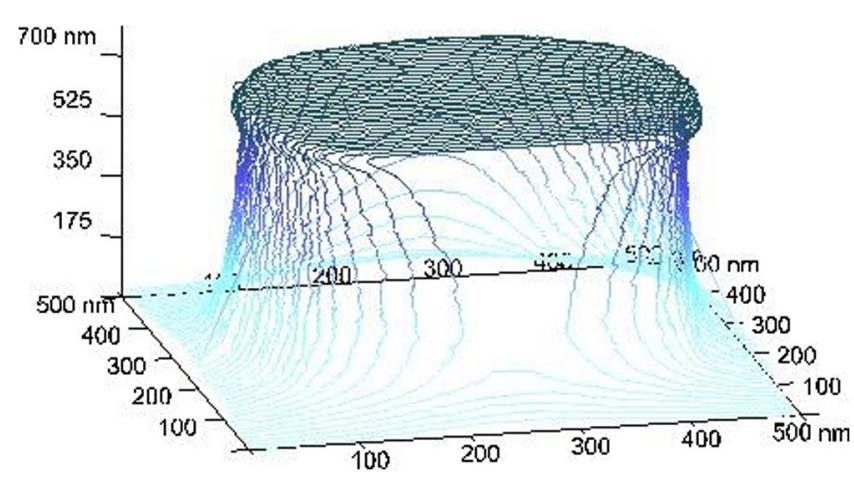

Fig. 5 Tip-on-tip image using a CDR120 tip on a CDR300 tip. The imaging stability is good. FDTA mode is used.

tip. Although a significant benefit of this for their purposes was the reduction of experimental complexity, it does also offer an end user the advantage of calibrating a linewidth standard, which can be retained for subsequent tip calibrations, as part of the self-consistency experiment. For CDAFM tips, the reduction of complexity comes with a loss of useful information: It is no longer possible to extract a self-consistent value for the VEH of the two CD tips. Despite this limitation, an experimental run of this type was performed using a CDR300 tip, a CDR120 tip, and a linewidth standard.

The tip-on-tip image of a CDR120 tip on a CDR300 tip is shown in Fig. 5. The results of the self-consistency experiment are shown in Table 3. In this case, the linewidth standard was a previously uncalibrated sample from the SCCDRM project. Generally, the results of this run were very good. The analyses of the self-consistency data using the native software and the off-line method are in very good agreement. Additionally, the agreement between the conventional and self-consistency methods is almost within the typical $1(k=2)$ to $1.3 \mathrm{~nm}(k=2)$ expanded uncertainty for the conventional method of tip calibration. For circumstances in which the only goal is TW calibration, this modified form of the self-consistency method is probably the best option. However, it is not applicable if there is also a need to determine the tip VEH.

Table 3 Comparison of conventional tip calibration with self-consistency method for CDR300, CDR120, and linewidth sample.

\begin{tabular}{|c|c|c|c|c|c|c|}
\hline & & \multicolumn{5}{|c|}{ Measurement method } \\
\hline \multicolumn{2}{|c|}{ Tip } & \multicolumn{2}{|c|}{$\begin{array}{l}\text { Conventional } \\
\text { (VPS/FSR) }\end{array}$} & \multicolumn{3}{|c|}{$\begin{array}{l}\text { Tip-imaging self- } \\
\text { consistency }\end{array}$} \\
\hline Type & Specifier & $\begin{array}{l}\text { TW } \\
(\mathrm{nm})\end{array}$ & $\begin{array}{l}\text { VEH } \\
(\mathrm{nm})\end{array}$ & $\begin{array}{l}\text { Analysis } \\
\text { software }\end{array}$ & $\begin{array}{l}\text { TW } \\
(\mathrm{nm})\end{array}$ & $\begin{array}{l}\text { VEH } \\
(\mathrm{nm})\end{array}$ \\
\hline \multirow[t]{2}{*}{ CDR300 } & A & 280.8 & $\mathrm{~N} / \mathrm{A}$ & Native & 279.1 & $\mathrm{~N} / \mathrm{A}$ \\
\hline & & & & Off-line & 279.4 & $\mathrm{~N} / \mathrm{A}$ \\
\hline \multirow[t]{2}{*}{ CDR120 } & B & 134.3 & $\mathrm{~N} / \mathrm{A}$ & Native & 132.9 & $\mathrm{~N} / \mathrm{A}$ \\
\hline & & & & Off-line & 133.0 & $\mathrm{~N} / \mathrm{A}$ \\
\hline $\begin{array}{l}\text { Linewidth } \\
\text { sample }\end{array}$ & C & 44.0 & $N / A$ & Native & 44.9 & $\mathrm{~N} / \mathrm{A}$ \\
\hline
\end{tabular}




\subsection{Dual Lateral Axis Extension of Technique}

The prior NIST work and the experiments described above involved the treatment of tip geometry in only a single lateral axis-the fast scan axis. From the metrology perspective, the fast scan axis is normally more important, and is therefore of primary interest. Additionally, unless cantilevers with tilt compensated tips are used, the profiles along slow scan axis, which is typically aligned with the long axis of the cantilever, will be influenced by tilt-related artifacts. For optimal use of data from both lateral axes, therefore, tilt-compensated tips are desirable.

Tip on tip imaging was further refined using smaller tips made from high-density carbon with cantilever tilt compensation. Prior attempts to use silicon tips $<50 \mathrm{~nm}$ were not successful, but it seemed plausible that the greater modulus of carbon relative to silicon would partially compensate for smaller size with respect to the resultant lateral stiffness. Nominal 25-nm carbon CD tips are commercially available. These tips are typically designated by the prefix electron beam deposition (EBD) as in: EBD-CDR25. An example of CDR25 on CDR25 tip imaging is shown in Fig. 6. Although noise and scanning instability are observed and present challenges, the image quality is sufficient to work with and to perform a self-consistency experiment.

In the prior work, the FDTA scanning mode was normally used. However, this mode is only available when the instrument $y$-axis, which also corresponds to the short axis of the cantilever, is selected as the fast scan axis. To treat both lateral axes in an equivalent manner, a fixed lateral dither was used instead for the CDR25 tip experiment. The fixed dither input voltage was set to $0 \mathrm{~V}$, corresponding to zero nominal dither, but in most cases the zero-input lateral motion of the tip is sufficient for the scanning control to operate. Using the same fixed input in both lateral axes, the nongeometrical contributions to the effective TW should be equivalent in both lateral axes.

Two complete sets of images for the self-consistency experiment were acquired using the same three CDR25EBD tips. The conventional tip characterization was also performed. For the $y$-axis fast scans, this involved imaging both a VPS and FSR sample-followed by a width check using the SCCDRM. The $x$-axis fast scans were calibrated directly using the SCCDRM. This reduced experimental steps, but also meant that conventional VEH measurements were not available for the $x$-axis fast scans. Table 4 shows both the

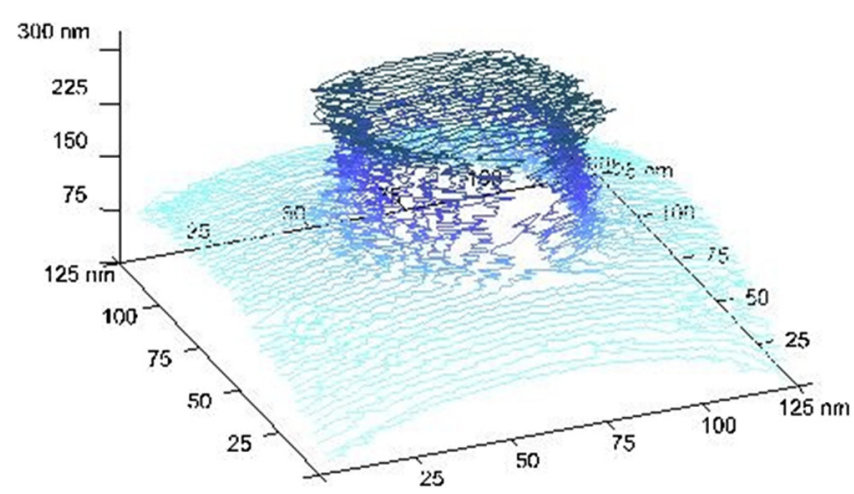

Fig. 6 Tip-on-tip image using a EBD-CDR25 tip on a EBD-CDR25 tip. The imaging stability is adequate for self-consistency data. A fixed lateral dither mode with $0 \mathrm{~V}$ was used.
Table 4 Results of conventional tip calibration and self-consistency method for EBD-CDR25 tips using the $x$-axis as the fast scan. Two self-consistency runs were performed, and each result from the second run is shown below the first.

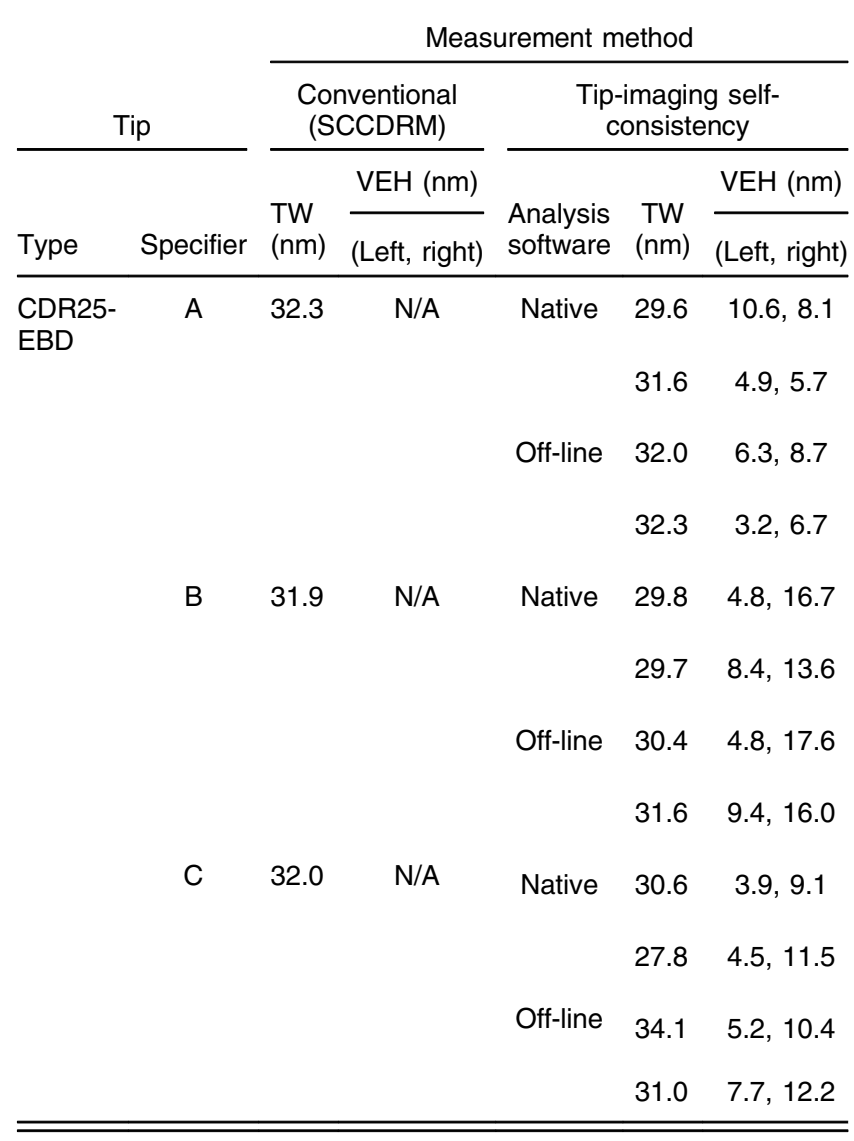

conventional and self-consistency results for the $x$-axis fast scan measurements. The y-axis fast scan results are shown in Table 5.

As a whole, the results shown in Tables 4 and 5 are favorable, particularly for the TW calibration and are approximately on par with or slightly surpassing the performance observed using CDR50S tips. In most cases, the agreement between the conventional and self-consistency TW values is at the 1- to 2-nm level. There is also usually similarly close agreement between the first and second self-consistency runs. The offline analysis and native analyses show agreement in the 1- to 3-nm range, with the offline results always larger. This is to be expected because the native analysis involves the waterfall function, which requires the selection of one scan line to represent the maximum diameter. If the tip shape in the $x-y$ scan plane is not an ideal circle, this constraint may introduce a small bias. As the offline analysis is based on the lateral extrema of the edges regardless of scan line, it is not subject to this bias. However, it is potentially more sensitive to edge noise, which is not negligible in these scans. A possible strategy for including more data in the analysis and reducing noise sensitivity is considered in the next section.

The VEH results generally show consistency to approximately the level of $5 \mathrm{~nm}$. In particular, the consistency between runs for the offline analysis was about $2 \mathrm{~nm}$. 
Table 5 Results of conventional tip calibration and self-consistency method for EBD-CDR25 tips using the y-axis as the fast scan. Two self-consistency runs were performed, and each result from the second run is shown below the first.

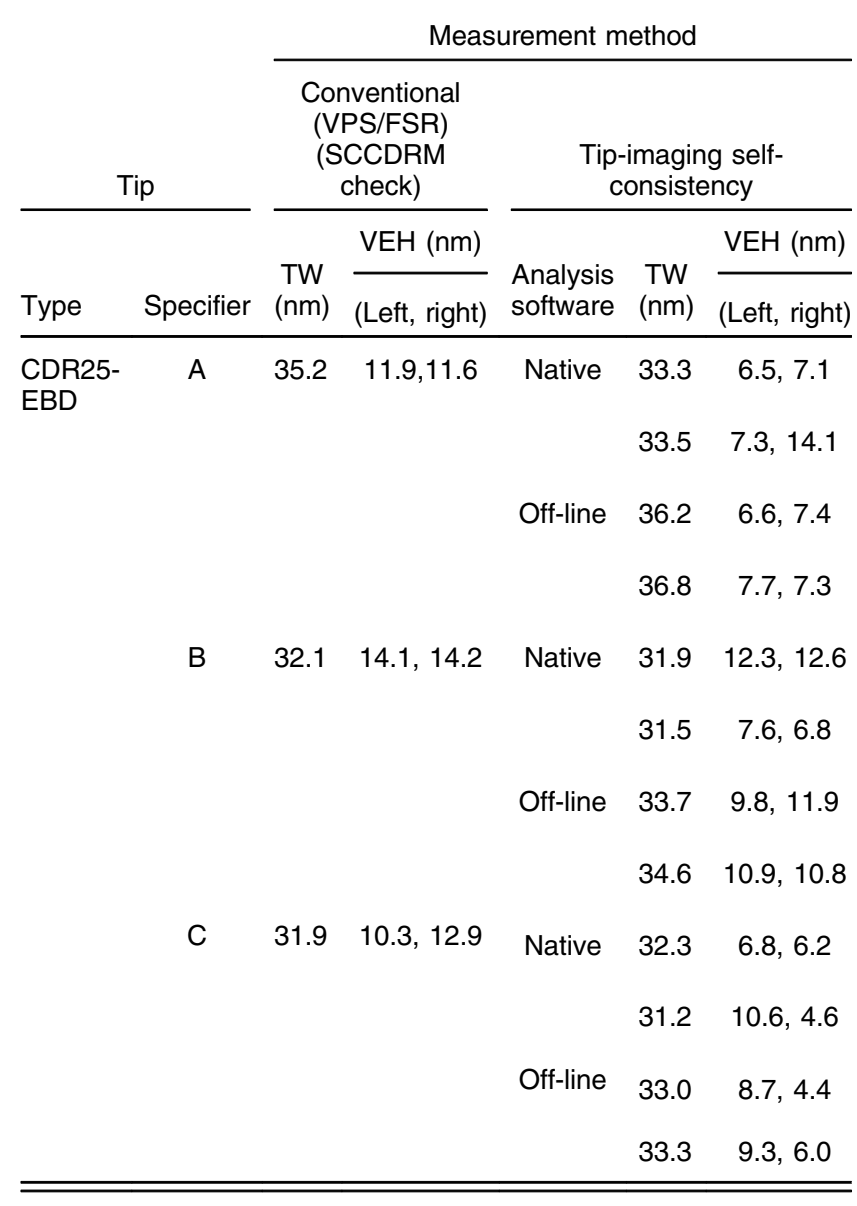

This performance is generally equivalent to or surpasses the results using other tip sizes. However, the goal of using selfconsistency results to establish a traceable calibration of VEH would require more stability in the results, and better agreement with both the native and conventional analyses and understanding the origin of any remaining offsets.

\subsection{Toward Planar/Contour Type Analysis}

The use of data in both lateral axes for self-consistency calibration is a step toward a complete geometrical representation of CD tips, but most of the information in the images is still not utilized. An additional step toward greater utilization of the images is to estimate a contour of the maximal tip shape in the $x-y$ scan plane.

A basic attempt at such planar analysis of tip shape was made using the "via" analysis function in the native software. This function, which is intended for the analysis of contact holes, allows extraction of the $x-y$ edge position at a certain height all around the perimeter of a feature. In this case, the feature analyzed is the tip-on-tip image, and height selected corresponds to the approximate sum of the VEH values for the two tips.

An example of the planar contour extracted from the run 2 tip B on tip A images (i.e., both $x$ - and $y$-axis fast scan) is

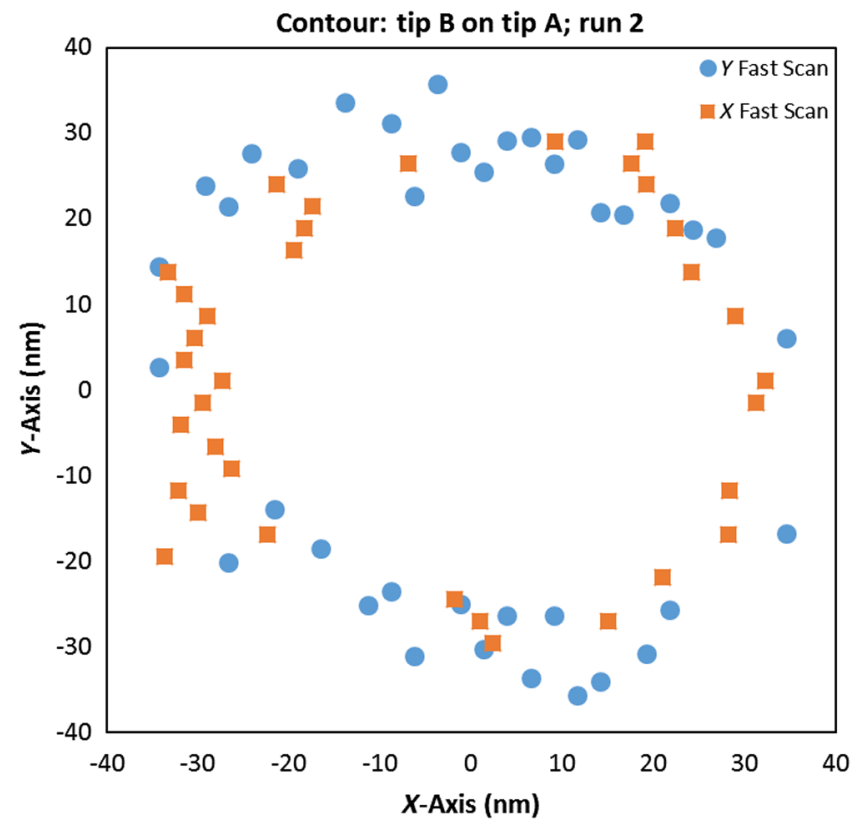

Fig. 7 Planar contour extracted from the run 2 images of tip B on tip A. Results obtained from both the $X$ - and $Y$-fast scan images are included.

shown in Fig. 7. The noise and outliers in edge location are significant, but are generally comparable with what is intuitively observed in the images as shown in Fig. 6. As expected, the performance using each axis as the fast scan is the worst near alignment with the orthogonal axis. This is true with respect to both the sampling density and the edge noise. In this example, the performance was surprisingly good and the edge outliers are less noticeable. In this case, none of the points is unambiguously excludable or clearly unphysical. But in some cases, points are observed that lie well outside the main "cluster" of results. When closely examined, however, these points are usually associated with bad scan lines-especially those containing tip "jumps." For most analysis purposes, it is thus reasonable to exclude such points from the contour. At the present stage of development, the exclusion process is performed manually by inspection, but it should be possible to develop a more automated analysis.

The extracted contours from both runs of tip C on tip B are shown in Fig. 8. In this plot, the $x$ - and $y$-axes fast scan results are included together for each run. It shows the overall reproducibility of the tip-on-tip contour. The most significant and unphysical outliers are easily identified and do not repeat between the two runs. However, there is still a nonnegligible level of edge detection noise-at about the 5-nm level. This is large enough that it must be considered in analysis and interpretation of the data.

The noise and variability of the tip-on-tip contour illustrate the challenging nature of the data. The analyses discussed in prior sections are based on finding the edge extrema along the fast scan axis. Conceptually, this is only strictly correct for sidewalls aligned with the slow scan axis or areas where the surface normal of the tip-sample contact area is aligned with the fast scan axis.

Additionally, the accuracy of the calculation also depends on the assumption that the extrema are representative of the 


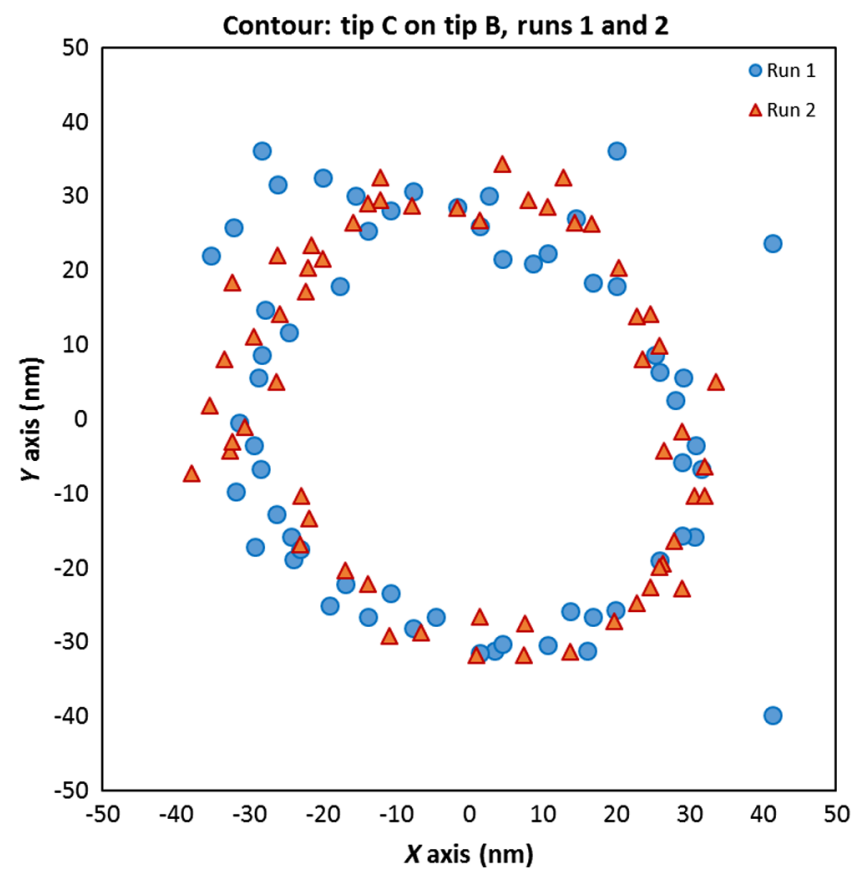

Fig. 8 Planar contours extracted from the run 1 and run 2 images of tip $C$ on tip B. Results obtained from four images (both the $X$ - and $Y$ fast scan from two runs) are included.

effective tip geometry rather than noise or jumps in the scan line. However, even after the clear outliers are excluded, the tip-on-tip contour in Fig. 8 shows that this assumption becomes questionable below the level of a few nanometers.

The question then arises as to how to best utilize the tipon-tip contour data. The self-consistency method is based on simple algebraic equations for three unknowns. However, it is only strictly applicable under certain conditions. The existence of a single well-defined TW metric, such as the radius of an ideal spherical tip, meets these conditions. A more general understanding of the constraints, however, requires some consideration of mathematical morphology.
The geometrical interaction of an AFM tip with a surface or in this case another tip is described by operations in mathematical morphology. ${ }^{11-13}$ Specifically, the geometry of the imaging tip dilates the features of the surface or target tip. As this operation is not always information preserving, the selfconsistency method may no longer be analytically exact, and the entire tip geometry may not be extractable. In terms of morphological operators, the requirement for the self-consistency method to be applicable to the tip-on-tip contours is that the tip contours are closed to each other. This means, for example, that a dilation of tip A by tip B, followed by an erosion of tip $B$, will result in recovery of the original tip A contour. A detailed discussion of the implications of this constraint for allowable tip contour geometries is outside the scope of this paper. In qualitative terms, it generally requires that the tip contours do not have asperities or notches with a radius of curvature significantly smaller than the overall radius of the tip contours. The analysis of tip on tip contours will be further investigated by NIST, but for purposes of this paper, the treatment will be restricted to the highly simplified cases of ideal circles and ellipses.

If a CD tip exhibited perfect rotational symmetry, its planar contour would be circular, and thus a single radius metric would be sufficient to characterize the tip shape in the $x-y$ scan plane. For CDR tips that are good condition, this assumption is often a reasonable approximation. This can be observed by comparing the conventionally calibrated TW values as shown in Tables 4 and 5, respectively. For tips $\mathrm{B}$ and $\mathrm{C}$, the observed difference in TW between the $x$ and $y$ axes is negligible and well under the $1 \mathrm{~nm}$ level of uncertainty. This is consistent with approximate rotational symmetry. Tip $A$, however, does exhibit a nonnegligible difference of $3 \mathrm{~nm}$ between the observed TW in $x$ and $y$ axes, respectively.

The potential applicability of the single radius model to tip on tip imaging and self-consistency calibration was tested for this overall dataset. These evaluations are shown in Table 6. A potential advantage of using a circular fit to

Table 6 Results of self-consistency analysis for EBD-CDR25 tips obtained using circular fits applied to the tip on tip contour data. Results using data from both runs are included, as well as values obtained using just the $x$ - or $y$-fast scan data and the $x / y$ data together.

\begin{tabular}{|c|c|c|c|c|c|c|c|}
\hline \multirow[b]{4}{*}{ Type } & \multirow[b]{4}{*}{ Specifier } & \multicolumn{6}{|c|}{ Measurement method } \\
\hline & & \multirow[t]{3}{*}{ Conventional (SCCDRM) } & \multicolumn{5}{|c|}{ Circular fit using contour From via analysis function } \\
\hline & & & & \multicolumn{4}{|c|}{ Self-consistent fit diameter $(\mathrm{nm})$} \\
\hline & & & & $X$-scan & $Y$-scan & $X$ and $Y$ & $X$ and $Y^{a}$ \\
\hline \multirow[t]{6}{*}{ CDR25-EBD } & A & $X$-axis: 32.3 & Run 1: & 33.4 & 29.7 & 31.4 & 32.4 \\
\hline & & $Y$-axis: 35.2 & Run 2: & 30.4 & 31.1 & 30.8 & 30.5 \\
\hline & B & $X$-axis: 31.9 & Run 1: & 28.5 & 32.4 & 30.6 & 29.6 \\
\hline & & $Y$-axis: 32.1 & Run 2: & 30.5 & 31.5 & 31.0 & 31.3 \\
\hline & C & $X$-axis: 32.0 & Run 1: & 33.4 & 31.8 & 32.5 & 30.1 \\
\hline & & $Y$-axis: 31.9 & Run 2: & 31.2 & 30.6 & 30.9 & 29.9 \\
\hline
\end{tabular}

${ }^{a}$ All outliers removed from contours. 
the tip-on-tip contours would be reduced sensitivity to noise in the detected edges. However, to the extent that the effective planar tip geometry deviates from ideal, a circular fit would be less accurate than locating the edge extrema. Based on the results shown in Table 6, it appears that the single radius model probably oversimplifies too much in most cases.

The reproducibility of the circular fit results is at approximately the same level as the edge extrema method. It is better in some cases but not in others. The level of consistency with the conventional TW calibration also varies but is not dramatically better. As tip A exhibits a detectable difference in the $x$ - and $y$-axes TWs using the conventional method, this is not surprising. Although tips B and C are closer to ideal, these results are influenced by the tip A results. Only in the case of three nearly ideal tips would the single radius approach be potentially applicable.

One interesting observation from Table 6 is a comparison of the third and fourth results columns on the right side. These columns show the results using circular fits to both the $x$ fast scan and $y$ fast scan data for each tip on tip combination. But as was seen in Fig. 8, there are usually unphysical outliers present in these contours. The calculations in Table 6, however, suggest that the influence of such outliers on circular fits is often not significant and is thus manageable. In most cases, the difference between the results with and without the outliers is $1 \mathrm{~nm}$ or less.

In the general case, as most CDR tips are expected to deviate from ideal, a circular fit is probably not a suitable approximation. The next logical step would be an elliptical fit, so this approach was also evaluated, and these results are shown in Table 7. Unfortunately, the general results of using elliptical fits are disappointing. At least for this dataset, the overall level agreement and consistency using elliptical fits are worse than using circular fits.

As can be seen in Table 7, perhaps the most disappointing observation is that the use of elliptical fits did not correctly capture the $x / y$ asymmetry of tip A that was observed using the conventional method. Additionally, the analysis introduced apparent asymmetry for tips B and C that was not observed using the conventional method. The level of agreement between run 1 and run 2 was not any better than with using circular fits. More surprisingly, the general consistency of the results seems to deteriorate when more outliers are excluded from the tip-on-tip contours.

The overall conclusion from this analysis is that neither elliptical nor circular fits to the CDR25 tip-on-tip contours demonstrated better performance than the prior method based on line-by-line analysis of the images. This is true both with respect to consistency with the conventional method and the apparent run-to-run reproducibility. Furthermore, the elliptical analysis was unable to correctly capture the $x / y$ tip asymmetry (or lack thereof), which was observed using the conventional method.

It thus seems that circular and elliptical fits are likely to be of minimal utility in the most general case due to edge detection noise and to geometrical deviations that are not well represented by either circles or ellipses. Specifically, for tips that exhibit nearly the same width in each axis - as tips B and C did in this work-the elliptical fit is not an improvement because a circular fit would be sufficient. In the presence of edge noise, the elliptical fit becomes slightly worse. Overall, the edge noise and deviations from idealized geometries were likely the primary reasons for the performance of the circular and elliptical fits relative to the line-by-line method. Further evaluation of fitting tip-on-tip contours is warranted, however, as these fits may be applicable in cases for which the $x$ and $y$ widths are in agreement or for which the contours are well described by ellipses.

Whether analysis using the tip-on-tip contours could have value in applications (e.g., measurement of in-plane corner radius) that require consideration of the tip geometry in the $x / y$ scan plane remains an open question. But for applications involving linear-type features, which require data using only a single fast-scan axis, there is no compelling reason to consider the tip-on-tip contours.

Table 7 Results of self-consistency analysis for EBD-CDR25 tips obtained using elliptical fits applied to the tip-on-tip contour data. Results using data from both runs are included, and the major and minor axes of the ellipse are used to determine the $x$-and $y$-axes TWs. For this analysis, the $x$ and $y$-fast scan data were always combined.

\begin{tabular}{|c|c|c|c|c|c|c|c|}
\hline \multirow[b]{4}{*}{ Type } & \multirow[b]{4}{*}{ Specifier } & \multicolumn{6}{|c|}{ Measurement method } \\
\hline & & \multirow[t]{3}{*}{ Conventional (SCCDRM) } & \multicolumn{5}{|c|}{ Elliptical fit using contour From via analysis function } \\
\hline & & & & \multicolumn{4}{|c|}{ Self-consistent fit axes (nm) } \\
\hline & & & & $X$-axis & $Y$-axis & $X$-axis $^{a}$ & $Y$-axis $^{\mathrm{a}}$ \\
\hline \multirow[t]{6}{*}{ CDR25-EBD } & $A$ & $X$-axis: 32.3 & Run 1: & 33.8 & 31.7 & 33.2 & 30.8 \\
\hline & & $Y$-axis: 35.2 & Run 2: & 32.3 & 29.9 & 30.4 & 30.7 \\
\hline & B & $X$-axis: 31.9 & Run 1: & 31.5 & 28.4 & 30.3 & 28.6 \\
\hline & & $Y$-axis: 32.1 & Run 2: & 33.5 & 30.5 & 35.3 & 29.7 \\
\hline & C & $X$-axis: 32.0 & Run 1: & 28.9 & 31.5 & 30.6 & 32.5 \\
\hline & & $Y$-axis: 31.9 & Run 2: & 28.1 & 31.4 & 29.5 & 31.8 \\
\hline
\end{tabular}

a Outliers removed from contours. 


\subsection{Assessing Relative Performance of Different Tip Types}

In the present work, three different tip types were evaluated: CDR50S, CDR140, and EBD-CDR25. A multitype and linewidth sample combination using a CDR120 and CDR300 tip along with a linewidth sample was also implemented. The prior NIST work ${ }^{7}$ involved three different tip types: CDR50C, CDR300, and CDR850. Additionally, the EBDCDR25 tips permitted a separate implementation for both lateral scan axes. Taken all together, this amounts to eight independent self-consistency experiments for TW, and seven for VEH as the implementation involving a linewidth sample does not allow for VEH extraction.

A cursory inspection of all the tip-on-tip images and the tables of self-consistency results reveals that the performance varies for different tip types. To be more quantitative than that, however, some form of performance metric is required, and different approaches are possible. For purposes of this discussion, a variability metric was defined by the following method: first, the difference between the observed maximum and minimum values of TW or VEH was calculated for each tip of each type. The second step was to average these values over all three tips (or LW sample) for each implementation. Note that the first step included all available values from both the conventional method and the self-consistency method using both the offline and native software analysis. It also included multiple runs that were available for the EBDCDR25 tips. Consequently, this value is a hybrid metric that includes reproducibility contributions, computational effects resulting from the analysis software, and any average offset between the self-consistency and conventional methods. Although other metrics are possible, the author regards this metric as a useful representation of the overall performance for purposes of comparing the implementations.

In Fig. 9, the width performance is shown as a function of tip type. The results shown include both those from the present and prior NIST work. Additionally, the performance among the implementations can be compared in two ways: the first is by the absolute value of the variability metric in nanometers, and the second is by a relative variability in which the variability metric is divided by the average value of the width for that implementation. This is potentially useful because comparing only the absolute values potentially understates the relative performance of larger tip sizes in the self-consistency implementation.

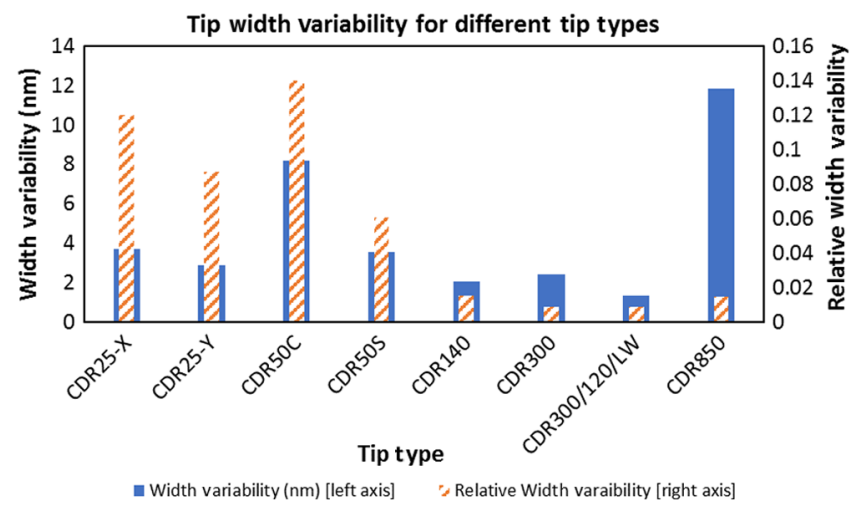

Fig. 9 Width variability metric as a function of tip type-including results from both present and prior NIST work.

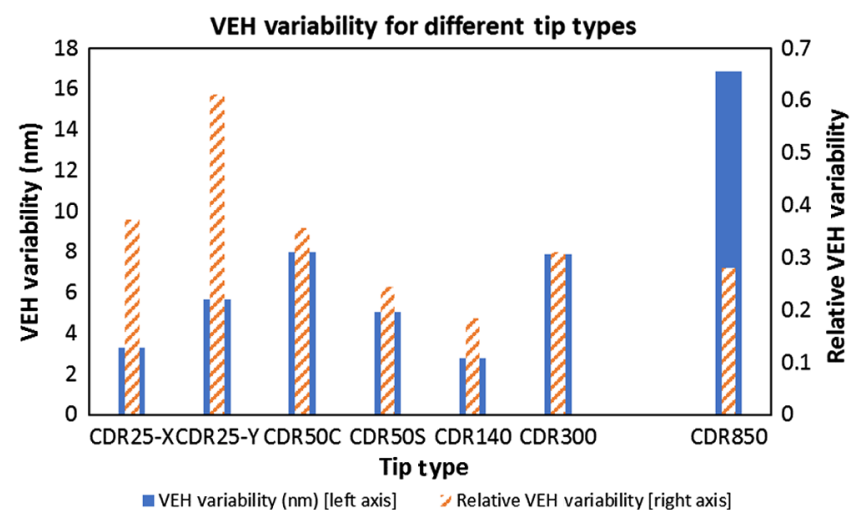

Fig. 10 VEH variability metric as a function of tip type-including results from both present and prior NIST work.

The visual representation of Fig. 9 is interesting, but there are ultimately few surprises to be found in comparing the performance of the different tip types. The results are consistent with the qualitative assessments given in the preceding sections. Optimum performance of the self-consistency width results is found in the range around CDR140 and CDR300 tips-with the linewidth sample run comparing very favorably. The figure also shows that the EBDCDR25 tips, despite being half the width, able to perform around the same level as the CDR50S tips. This is consistent with intuitive observation from the images, and it is probably due to the carbon composition and resultant lateral stiffness of these tips.

An analogous plot of performance for tip VEH as a function of tip type is shown in Fig. 10. Because the implementation using a CDR300, CDR120, and a linewidth sample does not yield a result for VEH, this run is not included in Fig. 10. As was the case for Fig. 9, the VEH performance among the implementations can be compared in two ways: the first is by the absolute value of the VEH variability metric in nanometers, and the second is by a relative variability in which the variability metric is divided by the average value of the VEH for that implementation. This is potentially useful because comparing only the absolute values potentially understates the relative performance of tips with larger values of VEH, and it also clearly emphasizes the challenges remaining with very small tips.

The visual representation of VEH performance shown in Fig. 10 is also interesting, but as was the case for the width performance there are ultimately few surprises to be found in comparing the VEH performance of the different tip types. The results are consistent with the qualitative assessments given in the preceding sections. Optimum performance of the self-consistency VEH results is found for CDR140 tips. The figure also emphasizes that the EBD-CDR25 tips perform well with respect to absolute VEH variability. However, because these tips tend to have smaller values of VEH in general, the figure also underscores the point that the relative VEH variability of the EBD-CDR25 tips is larger.

Ultimately, the quantitative variability analysis shown in Figs. 9 and 10 provides a foundation for the qualitative and intuitive inferences made from the tip-on-tip images and from a cursory inspection of the results in tables. There are no surprises, but the graphs clearly emphasize which tips have exhibited the optimum performance in self-consistency 
calibrations and for which tips there is room for more progress.

\section{Uncertainty Considerations}

As mentioned in Sec. 3, the typical expanded uncertainty for the conventional method of TW calibration is $1(k=2)$ to $1.3 \mathrm{~nm}(k=2)$. There is presently no traceable standard for $\mathrm{VEH}$, but the expanded uncertainty of the conventional method for $\mathrm{VEH}$ measurement is estimated to be $\sim 7 \mathrm{~nm}$ $(k=2)$. This estimate is obtained from the quadrature sum of two terms: a typical type A contribution is conservatively taken as $2 \mathrm{~nm}(k=1)$, and a type B estimate of the contribution arising from the characterizer edge radius (CER) is taken to be $2.9 \mathrm{~nm}(k=1)$. The general NIST approach to treatment of the CER contribution has been treated in a prior publication. ${ }^{14}$

Although the self-consistency method is still under development, the results of the multiple comparisons with the conventional method that have now been performed generally support a straightforward treatment of the self-consistency uncertainty. The self-consistency method does not require prior tip or width calibration. The terms on the right-hand side of Eq. (1) through Eq. (3) are all apparent widths and thus depend only upon instrument scale calibration. Consequently, in the ideal limit, the self-consistency method would not have sources of uncertainty pertaining to tip-ontip interaction and image dilation, but it would have uncertainty terms pertaining to scale calibration and a type A (statistical) contribution. Generally, the NIST results support this model, subject to the caveats discussed below.

The self-consistency results for TW obtained in different runs using different tip types are of variable image quality and numerical stability, but there have been no observations to suggest the presence of any bias related to an error source that cannot be treated within the basic model. A possible exception to this could be for the smallest and most flexible of tips to exhibit bending-related artifacts. To a first approximation, however, bending artifacts are expected to increase the effective TW relative to the geometrical width. With the assumption that a given tip exhibits the same effective width for both the conventional and self-consistent calibration, no significant bias would be expected in the results. This model could break down for the very smallest and most flexible tips, and NIST plans to investigate this further. However, the current results do not suggest any observable effect-particularly for the larger tips such as CDR120s. Note that the images using more flexible tips do exhibit a higher noise level, but the consequences of this are treated through the type A uncertainty analysis.

Although the self-consistency analysis for $\mathrm{VEH}$ is more challenging than for TW, there is still no indication of an error source that cannot be treated within a straightforward model. The tip-on-tip imaging noise and local surface asperities make both the definition of $\mathrm{VEH}$ and the implementation challenging. However, there are similar challenges in the conventional method, and the absence of a traceable standard further complicates the situation. NIST plans to investigate this further. Ultimately, it will probably be necessary to perform reference measurements using transmission electron microscopy (TEM) on both CD tips and characterizers to validate the uncertainty of VEH measurements.
The scale calibration uncertainties for the CD-AFM at NIST are on the order of $0.2 \%$ for the lateral axes and $0.5 \%$ for the vertical axis. Therefore, these contributions are negligible in most of the NIST tip-on-tip self-consistency experiments. The remaining uncertainty contributions are the statistical (type A) term and contributions pertaining to the measurand definition and the computational implementation of it.

The type A contribution requires multiple independent runs to evaluate. The number of runs available in this case is relatively small, which makes the evaluation challenging. In the context of ordinary measurements and provided that the system is functioning normally, this contribution is typically comparable from tip to tip and sample to sample over a reasonable window of tip and sample characteristics. It is thus often possible to use a general estimate of the expected magnitude of this contribution in the absence of sufficient data for evaluation. For CD-AFM width measurements, this general estimate is $0.5 \mathrm{~nm}(k=1)$, and the estimate for step height is typically smaller at $0.1 \mathrm{~nm}(k=1)$ to $0.2 \mathrm{~nm}(k=1)$. However, there is only partial applicability of these estimates to tip-on-tip imaging.

For CDR120 tips and larger, the estimate of $0.5 \mathrm{~nm}$ $(k=1)$ for apparent width measurements is approximately applicable, but for smaller tips a more realistic estimate would be $1(k=1)$ to $2 \mathrm{~nm}(k=1)$. However, the typical estimate for step heights would be a significant underestimate for VEH measurements. Conventional step height measurements involve the vertical separation of two horizontal surfaces. A significant number of data points are thus involved, and the sensitivity to noise spikes or small asperities is thus greatly reduced. VEH measurements involve very few data points and are thus very sensitive to vertical noise. Even for large tips, a realistic estimate would be at least $1 \mathrm{~nm}(k=1)$, with estimates as large as $3 \mathrm{~nm}(k=1)$ being more appropriate for the smallest tips.

The other important uncertainty contribution to consider would be a type B estimate pertaining to the ambiguity of the measurand definition and method of implementation. The definitions of width and VEH used in the NIST work correspond to the idealized definitions of these parameters. For nonpathological geometries, the implementation of these definitions is straightforward. Examples of pathological geometry, such as multiple flare apexes on one side of the tip, would often correspond to ambiguity or contextual dependence in the appropriate tip-sample interaction point for a given application. In the context of tip-on-tip imaging for typical tip shapes, there are few problems of this type. The greatest challenge with the implementation of the measurand definition is thus typically the lack of algorithm robustness against noise and anomalous jumps in the data. Future efforts will be made to improve robustness against noise and to better estimate this uncertainty. However, for present purposes this effect will be treated by its inclusion in the estimates of the type A term.

The bottom line is that the combined expanded uncertainty of the tip-on-TW results can be estimated as $\sim 1 \mathrm{~nm}$ $(k=2)$ for CDR120 and larger tips, but an estimate of $\sim 3 \mathrm{~nm}(k=2)$ is appropriate for CDR50 and smaller tips. For the tip-on-tip VEH results, the combined expanded uncertainty can be estimated as $2 \mathrm{~nm}(k=2)$ for CDR120 and larger tips. The estimate for smaller tips is $6 \mathrm{~nm}(k=2)$. 
Considering these uncertainty estimates, most of the width results in Table 1 through Table 5 are in good agreement among the methods and calculations. The results shown in Tables 2 and 3 are particularly encouraging with respect to immediate utility. The CDR140 results in Table 2 validate that a self-consistency TW calibration is possible with uncertainty approximately equivalent to the conventional method. However, the most immediately applicable results are the width results shown in Table 3 . This implementation using a linewidth standard in place of the third tip shows that a self-consistent width calibration experiment can be used to immediately yield a calibrated width standard in agreement with the conventional method and having an equivalent uncertainty. Although this conclusion would need to be further validated, probably using a TEM comparison, the results shown in Table 3 strongly suggest that this type of implementation of a self-consistent width calibration could surpass the performance of the conventional method.

The level of agreement among the VEH results shown in Table 1 thru Table 5 is not as good as the agreement for the width results, although most of the VEH results do agree within the estimated uncertainties. One notable issue, as was also the case in the prior NIST work, is that there are examples of significant differences between the VEH calculations using the native software and using the offline software. As these differences involve analysis of the same datasets, this highlights the challenge of implementing the idealized measurand definition of VEH on the actual tipon-tip images. This illustrates the need for further improvements in the VEH analysis and refined uncertainty estimates, as the highly idealized uncertainty model does not capture this. For example, the VEH results in Table 2 do not all agree within the uncertainties estimated for larger tips but do agree within the uncertainties estimated for smaller tips.

Additionally, the self-consistent VEH measurements seem more likely affected by potential physical sources of bias resulting from vertical noise and mechanical perturbations of the target tip during imaging. The results in Table 1 and especially Table 5 show indications of this, as some of the self-consistent values are near the estimated uncertainty limits or in marginal agreement. The bottom line is that while the self-consistent calibration of TW has been refined and demonstrated to be competitive with the conventional method, there is more work to be done before similar levels of confidence can be attained for the self-consistent calibration of VEH.

Ultimately, to refine and validate the uncertainty models for both self-consistent width and VEH, it will be necessary to acquire a significantly larger volume of data. The reliability of the estimated type A uncertainties will be significantly improved for a large number of runs, and this would help clarify the potential magnitude of any unknown sources of bias.

\section{Summary and Discussion}

The tip-on-tip imaging and self-consistency calibration method developed by NIST has been extended to tips $<50 \mathrm{~nm}$, and it has been demonstrated using both lateral axes for tips that have cantilever tilt compensation. Optimal performance relative to the conventional method was observed with larger tips such as CDR140s. Analogous to the modified approach of Eves and Green, ${ }^{6}$ in which a linewidth standard is substituted for the third tip, an experiment using CDR120, CDR300, and a linewidth standard was also performed. Although this approach does not allow VEH calibration, it is probably the most readily implemented by an end user of CD-AFM and offers immediately useful results in yielding a known width value for a linewidth structure.

The inclusion of data from using both lateral axes for the fast scan works best when using tips with cantilever tilt compensation such as the EBD-CDR25 tips used in this work. In addition to a self-consistency analysis based on line by line analysis of the tip-on-tip images, a contour-type analysis of the tip-on-tip images was performed. Both circular and ellipsoidal fits to the tip-on-tip contours were evaluated. For measurements of linear type features, however, there is probably no advantage to analyzing the tip-on-tip contours. Indeed, as the effective TW along a given axis is determined by the edge extrema along that axis, the width determined from the lineby-line analysis would typically be close to the physically relevant definition of TW, whereas the edge defined by a circular or ellipsoidal fit would only correspond to the physically relevant definition of the TW for geometries that are very close to an ideal circle or ellipse.

It is also probable, however, that the tip-on-tip contour analysis will have value for such applications as contour metrology or measurement of contact holes, because analysis of the tip geometry in the $x / y$ scan plane would be important to such measurements. This will be area of future investigation by NIST.

The imaging stability challenges generally experienced using smaller tips, particularly those $<100 \mathrm{~nm}$ in width, are thought to be due primarily to higher lateral tip compliance and mechanical perturbations during imaging. The EBDCDR25 (nominal $25 \mathrm{~nm}$ ) tips used in this work were comprised of carbon instead of silicon, and this material difference appeared to generate sufficiently high lateral stiffness despite the narrow width. The other means of improving stiffness would be to reduce the effective or working lengths of the tips. Although this would also reduce the general applicability of such tips, NIST intends to try experiments using custom tips with very short working lengths - such as $50 \mathrm{~nm}$ - to further test the limits of the tip-on-tip self-consistency method.

Finally, it should be noted that the self-consistency method of tip calibration is still generally too cumbersome for routine use on a production tool, especially in a fully inline environment. In this context, high levels of automation and minimal user intervention are very important metrics. At the present time, the self-consistency method is essentially manual and its challenges require considerable judgement and action by the user. Examples of this include the requirement for manual inversion of the tip orientation, the difficulty of navigation, and the typically resultant need for multiple engages. Although it will likely be possible to further refine the method in some respects, it is probably not suitable for in-line use by a minimally experienced operator. However, for those CD-AFMs being utilized in a process-intermittent role or in an off-line laboratory environment by experienced operators, the method may be suitable for occasional use.

\section{Acknowledgments}

This work was supported by the Microsystems and Nanotechnology Division of the NIST Physical Measurement 
Laboratory. Certain commercial equipment is identified in this paper to adequately describe the experimental procedure. Such identification does not imply recommendation or endorsement by the National Institute of Standards and Technology nor does it imply that the equipment identified is necessarily the best available for the purpose.

\section{References}

1. Y. Martin and H. K. Wickramasinghe, "Method for imaging sidewalls by atomic force microscopy," Appl. Phys. Lett. 64, 2498-2500 (1994).

2. M. Tortonese et al., "Sub-50-nm isolated line and trench width artifacts for CD metrology," Proc. SPIE 5375, 647-656 (2004).

3. R. G. Dixson et al., "Traceable calibration of critical-dimension atomic force microscope linewidth measurements with nanometer uncertainty," J. Vac. Sci. Technol. B 23, 3028-3032 (2005).

4. G. Dai et al., "Development and characterization of a new line width reference material," Meas. Sci. Technol. 26, 115006 (2015).

5. S. J. Cho et al., "Three dimensional imaging of undercut and sidewall structures by atomic force microscopy," Rev. Sci. Instrum. 82, 023707 (2011).

6. T.-G. Kim et al., "In-line critical dimension and sidewall roughness metrology study for compound nanostructure process control by in-line 3D atomic force microscope," ECS Trans. 75, 761-767 (2016).

7. R. Dixson, "Tip on tip imaging and self-consistent calibration using critical dimension atomic force microscopy," J. Micro/Nanolith MEMS MOEMS 16, 024005 (2017).
8. B. J. Eves and R. G. Green, "Self-consistent determination of line-width and probe shape using atomic force microscopy," Meas. Sci. Technol. 24, 085401 (2013).

9. R. Dixson et al., "Traceable calibration of a critical dimension atomic force microscope," J. Micro/Nanolith. MEMS MOEMS 11, 011006 (2012).

10. M. Cresswell et al., "RM8111: development of a prototype linewidth standard," J. Res. Natl. Inst. Stand. Technol. 111, 187-203 (2006).

11. J. S. Villarrubia, "Algorithms for scanned probe microscope image simulation, surface reconstruction, and tip estimation," J. Res. Natl. Inst. Stand. Technol. 102, 425-454 (1997).

12. X. Qian and J. S. Villarrubia, "General three-dimensional image simulation and surface reconstruction in scanning probe microscopy using a dexel representation," Ultramicroscopy 108, 29-42 (2007).

13. F. Tian, X. Qian, and J. S. Villarrubia, "Blind estimation of general tip shape in AFM imaging," Ultramicroscopy 109, 44-53 (2008).

14. R. Dixson et al., "Interaction of higher order tip effects in CD-AFM width metrology," J. Vac. Sci. Technol. B 33, 031806 (2015).

Ronald Dixson is a physicist in the Physical Measurement Laboratory (PML) of the National Institute of Standards and Technology (NIST). His current research interests are calibration methods, traceability, and uncertainty analysis in atomic force microscope (AFM) dimensional metrology-including the NIST traceable AFM (T-AFM) project and metrology applications of critical dimension AFM (CD-AFM). He holds a PhD in physics and is a member of SPIE and the American Physical Society. 